\title{
Investigating the STING Pathway to Explain Mechanisms of BCG Failures in Non-Muscle Invasive Bladder Cancer: Prognostic and Therapeutic Implications
}

\author{
Madhuri Koti $^{\mathrm{a}, \mathrm{b}, \mathrm{c}, \mathrm{d}, *}$, Stephen Chenard ${ }^{\mathrm{a}, \mathrm{c}, \mathrm{d}}$, Sarah Nersesian ${ }^{\mathrm{e}}$, Thiago Vidotto ${ }^{\mathrm{f}}$, \\ Alvaro Morales ${ }^{\mathrm{d}}$ and D. Robert Siemens $\mathrm{s}^{\mathrm{a}, \mathrm{c}, \mathrm{d}}$ \\ ${ }^{a}$ Department of Biomedical and Molecular Sciences, Queen's University, Kingston, Canada \\ ${ }^{\mathrm{b}}$ Department of Obstetrics and Gynecology, Kingston Health Sciences Center, Queen's University, \\ Kingston, Canada \\ 'Cancer Biology and Genetics, Queen's Cancer Research Institute, Queen's University, Kingston, Canada \\ ${ }^{\mathrm{d}}$ Department of Urology, Kingston Health Sciences Center, Queen's University, Kingston, Canada \\ ${ }^{\mathrm{e}}$ School of Medicine, Dalhousie University, Halifax, Canada \\ ${ }^{\mathrm{f}}$ Department of Pathology, Johns Hopkins University School of Medicine, Baltimore, MD, USA
}

\begin{abstract}
.
Background: Intravesical Bacillus Calmette Guerin (BCG) has been the gold standard immunotherapy to treat high risk non-muscle invasive bladder cancer (NMIBC) for over 40 years. Attenuation of Mycobacterium bovis for clinical use as BCG results in loss of its ability to activate the "Stimulator of Interferon Genes" (STING) pathway and potentially limits local anti-tumor immune activity and subsequent BCG responsiveness due to reduced induction of the immune cell recruiting chemokines primarily, CXCL10. We conducted the current study to determine the potential of STING pathway agonist in synergizing with BCG to enhance chemokine induction.

Methods: The TICE strain of BCG (OncoTICE) was used in combination with STING agonist to determine STING pathway activation and CXCL10 production in THP-1 monocytic cell line, THP-1 defNLRP3, THP-1 dual STING knock out cells, RT112 bladder cancer cells and primary bladder epithelial cells. NanoString platform-based gene expression profiling and multiplex cytokine analysis were performed to determine induction of interferon associated genes and secreted cytokines.

Results: Activation of cytosolic pattern recognition receptor and downstream IFN1 pathways demonstrated synergistic activation of STING pathway enhanced BCG induced inflammasome and STING pathway gene expression in monocytes and bladder cancer cells. The significant differences in CXCL10, CCL5, IL-8 and MIP-1a/1b amongst the knock-out cell lines confirm the convergence of these pathways following combination treatment with BCG and STING agonist.

Conclusions: Findings from our study are the first evidence indicating that STING pathway activators are promising new innate immune modulators with a potential to synergize with BCG therapy in the treatment of NMIBC.
\end{abstract}

Keywords: BCG, STING agonist, immunotherapy, NMIBC, interferon, CXCL10, CD8+ TIL

\section{INTRODUCTION}

*Correspondence to: Madhuri Koti, DVM, MVSc, PhD, Department of Biomedical and Molecular Sciences and Obstetrics and Gynecology, Queen's University, Kingston, K7L3N6, ON, Canada. E-mail: kotim@queensu.ca.
Intravesical instillation of BCG has demonstrable benefit, however, some patients exhibit sub-optimal responses, with many suffering from recurrence and 
progression to secondary muscle invasive disease [1]. Features associated with response to BCG treatment include patient age, sex/gender, and prior BCG exposure [2]. Despite being used as the primary treatment for high-risk non-muscle invasive bladder cancer (NMIBC) for over 40 years, the mechanisms underlying the anti-cancer therapeutic effects of BCG in bladder cancer patients have yet to be fully established. In the context of tuberculosis however, the mechanism of action of BCG has been well demonstrated, including the uptake of the mycobacterium by macrophages, leading to the production of immune cell recruiting chemokines [3].

BCG vaccination is used as the most reliable prophylactic approach for tuberculosis across the globe. While most studies indicate that macrophages and monocytes are the predominant cell types where mycobacteria reside and multiply, some evidence has pointed towards a more dominant role of dendritic cells, which are more potent interferon producers (DCs) [4]. Regardless of the cell type, the intracellular location of infectious Mycobacterium tuberculosis $(M t b)$ leads to the release of bacterial DNA in the cytosol of the infected cell through the early secretory antigenic target (ESAT-6) secretion system 1 (ESX-1 secretion system located on 'region of differentiation 1'/RD1) [3], which is also critical to the delivery of $M t b$ mRNA into the cytosol [5]. Infection with mycobacterial strains with intact ESX-1 system (or SecA2 system) leads to IFN- $\beta$ secretion. This process occurs through cross-talks between the AIM2/NLRP3/CASP1 inflammasome, cGASSTING/IRF3 and the RIG/MAVS/IRF7 cytosolic nucleic acid sensing pathways [3,6].

Although attenuation is critical to reduce pathogenicity of the bacterium, in the case of M.bovis, this process removes the bacterium's ability to activate the cGAS-STING/IRF3 and the RIG/MAVS/IRF7 pathways. Both of these pathways normally complement IFN1-induced chemokine production. Therefore BCG, which lacks the ESX-1 secretion system, can only lead to the activation of Type I IFN (IFN1) responses via the AIM2/NLRP3/CASP1 inflammasome pathway. This attenuated chemokine induction may be responsible for reduced recruitment of CD4+ and CD8+ T cells [3, 6]. Exploiting these features, a recent report demonstrated that mucosal delivery of STING pathway activating ligands provided superior protection to $M t b$ challenge than BCG in a mouse model $[6,7]$. This enhanced protection occurred via recruitment of CXCR3 + IFN- $\gamma$ producing $T$ cells and was IFN1 independent.
In the case of bladder cancer, it is thus plausible that local administration of BCG lacking the ESX-1 system results in some activation of IFN and downstream induction of $\mathrm{T}$ cell attracting chemokines such as CXCL10. However, the magnitude of this activation does not suffice to produce the anti-tumour immune cell recruitment necessary for the elimination of existing tumours, nor does it prevent recurrence or progression after BCG treatment (Fig. 1).

Recent advances in the field of immunomodulatory therapies, have introduced a novel class of drugs called "Stimulator of Interferon Genes (STING) ligands/agonists"[8]. The STING pathway is primarily activated via cytosolic DNA and also intersects with multiple cellular cytosolic nucleic acid sensing pathways that ultimately lead to IFN induced chemokine induction and therefore has recently re-gained attention in immunomodulatory cancer therapeutics [9-11]. In pre-clinical immunocompetent models of solid cancers, we and others have shown that STING agonists function via enhancing the antigen cross priming potential of dendritic cells and macrophages, eventually leading to enhanced IFN response and production of immune cell recruiting chemokines, CXCL9/10/11, and associated activated CD8+ T cell recruitment $[9,11,12]$.

The sub-optimal control of higher risk NMIBC with intravesical agents as well as global shortages of BCG leading to reduced dosing for bladder cancer patients, further strengthens the rationale for the development of alternate immune adjuvants that can enhance endogenous IFNI activation and potentiate the efficacy of BCG. Based on the evidence that inflammasome activation is the sole mechanism of IFN induction in macrophages infected with BCG mycobacterial strain, in the current study we investigated whether activation of the STING pathway, simultaneous to treatment with BCG increases production of the key immune cell recruiting chemokine, CXCL10 and others, in primary bladder epithelial cells, cancer cells and immune cells.

\section{MATERIALS AND METHODS}

In this study we used three monocytic cell lines; THP-1, THP1-defNLRP3 cells (lacking NLRP3 gene required for AIM2/NLRP3/CASP1 inflammasome pathway activation, this pathway being critical to BCG mechanism of action) and THP1Dual $^{\text {TM }}$ KO-STING cells (lacking TMEM173 gene that encodes of STING). THP-1 cells (ATCC) were 

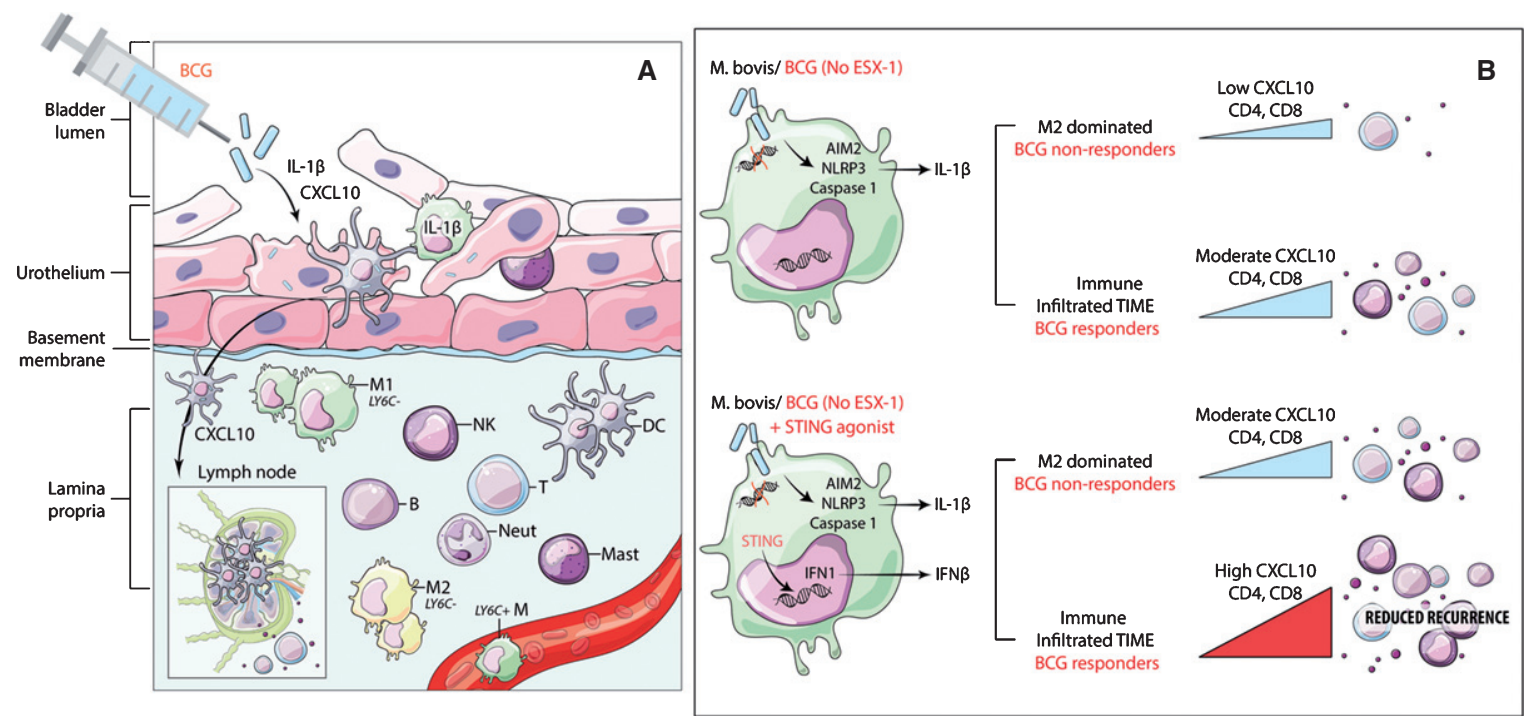

Fig. 1. Schematic showing conceptual model of potential mode of BCG action and the expected effect of STING pathway activation to increase chemokine secretion and immune cell recruitment. A) Proposed model for the mode of action of BCG in bladder cancer showing the critical need for STING pathway in synergistically contributing to CXCL10 production. Following intra-vesical instillation, BCG in the bladder lumen is internalized by the epithelial lining, which is constituted by untransformed urothelial cells, tissue resident cell types including macrophages, DCs and mast cells. Antigen cross presentation via DCs and macrophages presumably occurs either through trafficking via local lymph nodes or cross-priming to pre-existing T cells in the local microenvironment. Under the influence of chemokine (e.g. CXCL10) gradients, immune cell trafficking to the site of inflammation restricts tumour recurrence and progression. B) Macrophage with intracellular BCG showing no activation of STING pathway and a modest increase in CXCL10 secretion and immune cell recruitment under an M2 macrophage dominated environment. Addition of STING agonist to BCG treatment could lead to significant increase in CXCL10 and immune cell recruitment to reduce cancer recurrence.

maintained in RPMI-1640 with $10 \%$ heat-inactivated fetal bovine serum (FBS) and penicillin/streptomycin $(100 \mu \mathrm{g} / \mathrm{ml})$ as recommended. The THP1-defNLRP3 cells (Invivogen) and THP1-Dual ${ }^{\mathrm{TM}}$ KO-STING cells (Invivogen) were maintained in RPMI 1640, $2 \mathrm{mM}$ L-glutamine, $25 \mathrm{mM}$ HEPES, 10\% FBS, $100 \mu \mathrm{g} / \mathrm{ml}$ Normocin ${ }^{\mathrm{TM}}$, penicillin/streptomycin $(100 \mu \mathrm{g} / \mathrm{ml})$. Blasticidin and zeocin were added to the growth media in the knockout derivatives of THP-1, at every 2-3 passages. Primary bladder epithelial cells (PBEC) were purchased from ATCC and maintained in Prostate Epithelial Basal Medium $\left(\mathrm{ATCC}^{\circledR}\right.$ No. PCS-440-030) supplemented with Corneal Epithelial Growth Kit (ATCC ${ }^{\circledR}$ No. PCS-700-040). The bladder cancer cell line RT112 was obtained from Sigma Aldrich and maintained in Eagle's Modified Essential Medium with $10 \%$ FBS.

\section{NanoString based IFN pathway gene expression analysis}

To measure gene expression changes in STING pathway associated genes, post treatment with BCG alone or in combination with STING agonist, a custom NanoString gene panel comprising of genes associated with activation of cytosolic pattern recognition receptors (PRR) and downstream IFN1 pathways (Supplementary Table 1) was used. BCG (OncoTICE, Merck) was used at the previously described dose of $8 \times 10^{4} \mathrm{CFU}$ [13]. STING agonist (2'3'-c-di-AM (PS) 2 (Rp, Rp), Invivogen) was used at a concentration of $2 \mu \mathrm{g} / \mathrm{ml}$. Total RNA was isolated from all cell lines (as indicated) at $6 \mathrm{~h}$ post treatment with BCG or STING agonist or both as described above, using the total RNA Purification Kit (RNeasy mini kit, Qiagen Inc.) as per the manufacturer's instructions. RNA concentration and purity were estimated on a NanoDrop ND-100 spectrophotometer (NanoDrop Technologies, Wilmington, DE, USA). $150 \mathrm{ng}$ of total RNA from each tumour sample was subjected to digital multiplexed profiling, using the pre-built PRR gene panel with 5 housekeeping controls (NanoString Technologies Inc.) as per our previously established protocols [14, 15]. Normalization of raw data was performed using the nSolver software 3.0 (NanoString Technologies, Seattle, WA). The raw NanoString counts were initially subjected to normalization for all target RNAs 
in all samples based on built-in positive controls. This step accounts for inter-sample and experimental variation such as hybridization efficiency and post-hybridization processing. The geometric mean of each control was calculated to indicate the overall assay efficiency. The housekeeping genes were used for mRNA content normalization. Differentially expressed genes between different treatment groups were determined using Kruskal-Wallis test.

\section{Multiplex cytokine analysis}

To determine the effect of BCG with or without addition of STING agonist on the levels of secreted IFN induced cytokines, all the above described cell lines including non-malignant primary bladder epithelial cells, were seeded in appropriate growth media at the indicated densities. BCG (OncoTICE, Merck) was used at the previously described dose of $8 \times 10^{4} \mathrm{CFU}$ [13]. STING agonist (2'3'-c-di-AM (PS) $2(R p, R p)$, Invivogen) was used at a concentration of $2 \mu \mathrm{g} / \mathrm{ml}$. Both treatments were conducted as single agents and in combination under reduced serum $(2 \%)$ conditions. No treatment media controls were also included. Following $24 \mathrm{~h}$ incubation at $37 \mathrm{C}$ post stimulation, supernatants were collected and subjected to Human Cytokine/Chemokine Array 42-Plex with IL-18 (HD42) based multiplex cytokine analysis using the Luminex ${ }^{\mathrm{TM}} 100$ system (Eve Technologies, Calgary, AB, Canada). Differences between the treatment groups were tested using a two-way ANOVA (Graphpad Prism 9.0, Inc.). A $p$-value $<0.05$ was considered significant.

\section{RESULTS}

Synergistic activation of STING pathway enhances the effect of BCG induced IFN1 pathway genes in monocytes and bladder cancer cells.

In THP-1 cells with intact NLRP3 and STING pathways, addition of STING agonist led to increased levels of AIM2, CCL4, CXCL10, DDX58, IFI16, IFI44, IFIT2. IFIT3, IFITM3, IFNB1, IL6, IRF7, ISG15, MX1, OAS1A, OAS3, OASL1, PSMB9, STAT1 and USP18 compared to either BCG or STING agonist treatment alone (Fig. 2). At the $6 \mathrm{~h}$ time point, only IL1B and IRF1 gene expression was statistically significant altered (Fig. 2). In line with the previously confirmed role of the NLRP3 inflammasome in BCG mediated IFN activation, we observed reduced expression of the genes mentioned above, in the NLRP3 KO cells post treatment with BCG + STING agonist (Fig. 3). Similarly, the critical role of STING pathway in potent induction of IFN1/chemokine genes was confirmed by the significantly reduced expression of STING pathway genes described above excluding CCL4 and IL1B (Fig. 3). Compared to BCG alone, a higher expression of IFN1 genes was noted in the THP1-defNLRP3 cells treated with BCG + STING agonist (Fig. 3a). Indeed, the decreased expression of IFN1 genes in THP1-Dual ${ }^{\text {TM }}$ KO-STING cells confirmed benefits and synergism of inducing the STING pathway in combination with BCG (Fig. 3b). Similar to the monocytic cell line, the RT112 bladder cancer cell line also showed significantly higher expression of CXCL10, DDX58, IFI16, IFI44, IFIH1, IFIT1, IFIT2, IFIT3, IFITM3, ISG15, MX1, OAS3, OASL1, STAT1 and USP18 genes post

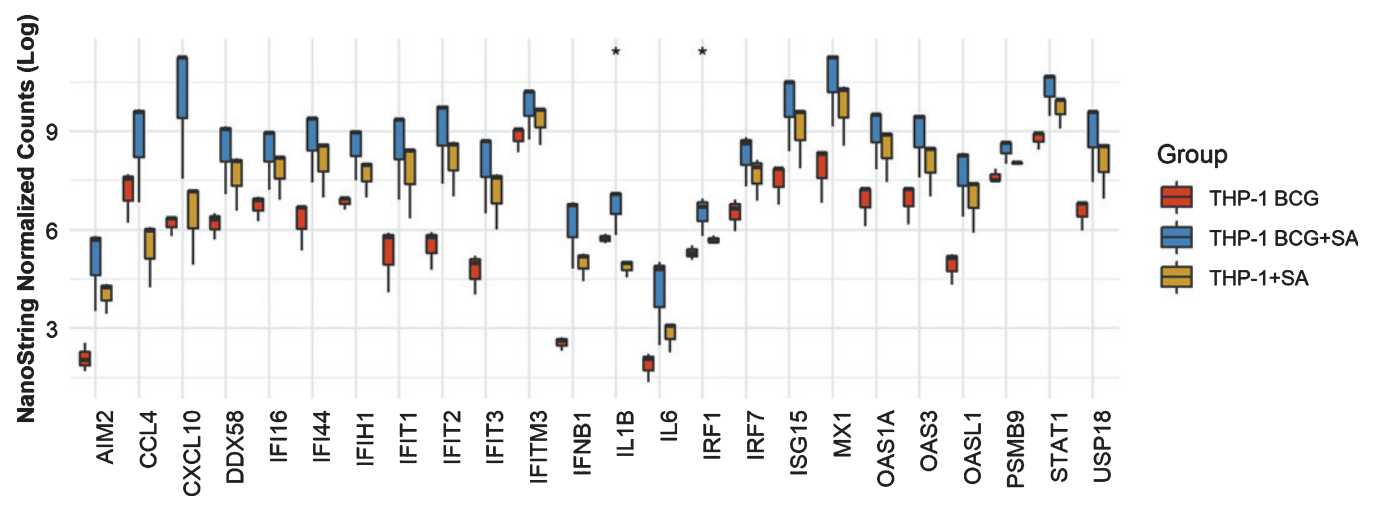

Fig. 2. Synergistic effect of BCG and STING agonist combination on induction of IFN1 genes in THP-1 cells. NanoString platform-based gene expression profiling of THP-1 cells treated with BCG and BCG+STING agonist. A custom IFN gene panel was used to determine expression changes at $6 \mathrm{~h}$ post treatment. NanoString data was normalized using nSolver software. RNA isolated from three independent experiments was used for gene expression analysis. Kruskal-Wallis test was applied to determine statistically significant $(* p<0.05)$ expression differences between treatments. 
A

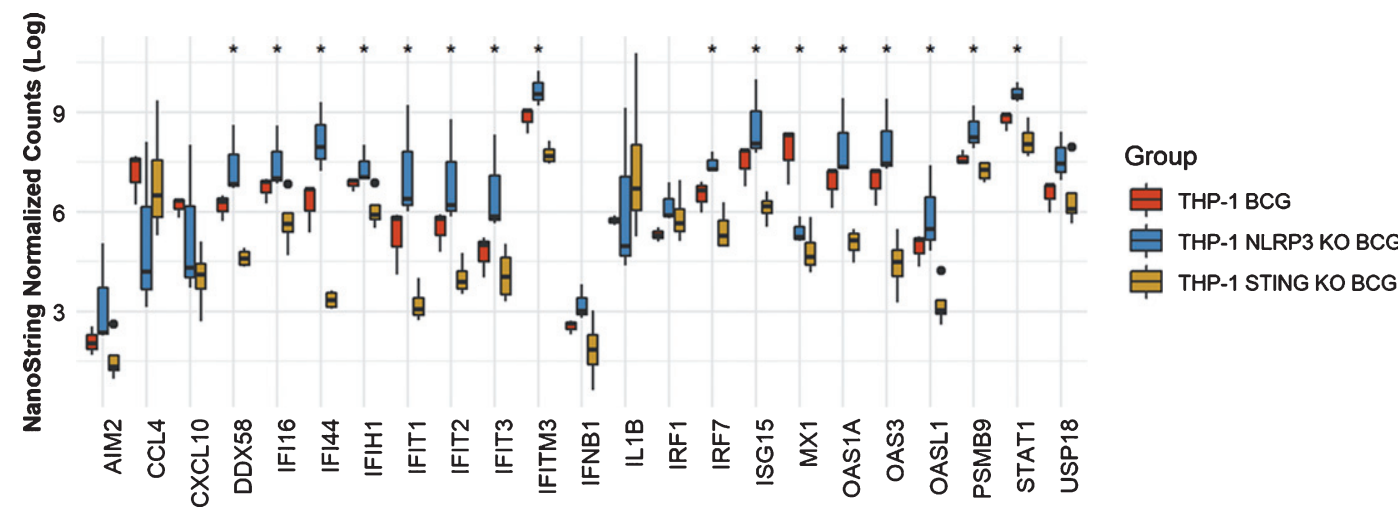

B

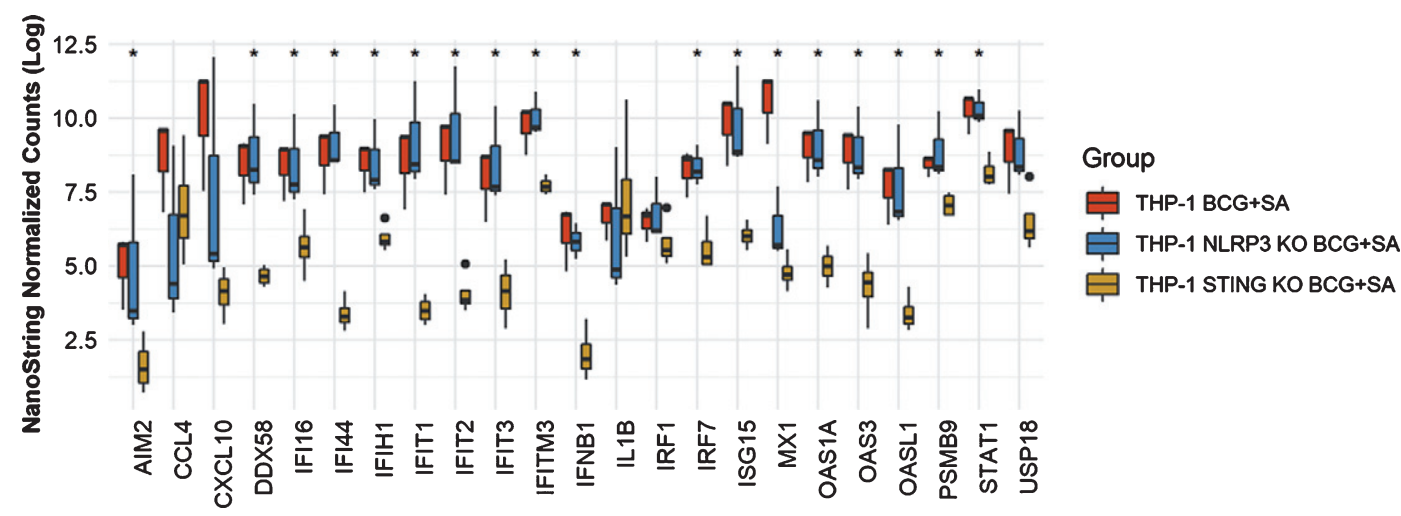

Fig. 3. Role of NLRP3 and STING pathways in induction of IFN1 genes in response to treatment with BCG and STING agonist combination. NanoString platform-based gene expression profiling of THP-1def NLPR3 and THP-1 dual STING KO cells treated with BCG and $\mathrm{BCG}+\mathrm{STING}$ agonist. A custom IFN gene panel was used to determine expression changes at $6 \mathrm{~h}$ post treatment. RNA isolated from three independent experiments was used for gene expression analysis. NanoString data was normalized using nSolver software. Kruskal-Wallis test was applied to determine statistically significant $\left({ }^{*} p<0.05\right)$ expression differences between treatments.

combination treatment with BCG and STING agonist compared to BCG treatment alone ( $p<0.05$; Fig. 4$)$.

Addition of STING agonist in combination with $B C G$ treatment significantly increases secreted levels of chemokines CXCL10, CCL5, IL-8 and MIP-1a/lb in THP-1 monocytic cell line

To determine the effect of combination treatment with BCG and STING agonist on secreted levels of chemokines key to immune cell recruitment, THP-1 and its derivative cell lines were treated with BCG with or without STING agonist. Secreted cytokines/chemokine levels were measured $24 \mathrm{~h}$ post treatment. BCG treatment led to significantly increased levels of CXCL10, CCL5, IL-8, MIP-1a and MIP-1b in THP-1 cells compared to THP-1 defNLRP3 and THP1-Dual ${ }^{\mathrm{TM}}$ KO-STING cells. Except CCL5, all four cytokines were minimally expressed in THP-1 defNLRP3 cells (Fig. 5a). Treatment with BCG+STING agonist led to signifi- cantly higher increase in levels of CXCL10, IL-1RA, CCL5, IL-8, MIP-1a and MIP-1b in the THP-1 cells compared to either BCG or STING agonist alone (Fig. 5b). In the STING agonist treated cells, significantly higher levels of CXCL10 were observed in the THP-1 defNLRP3 cells. IL-8 levels were highest in the THP1-Dual ${ }^{\mathrm{TM}} \mathrm{KO}-\mathrm{STING}$ cells post STING agonist treatment (Fig. 5c). IL-1RA levels were significantly higher in the THP-1 defNLRP3 cells compared to the other two cell lines, indicating the role of STING pathway in IL-RA secretion. The significant differences in CXCL10 and IL-8 amongst the three cell lines confirms the convergence of these pathways post combination of BCG with STING agonist.

\section{Primary bladder epithelial cells secrete CXCL10 in response to BCG and STING agonist treatment}

Since the bladder mucosa is primarily dominated by non-malignant epithelial cells at the time of BCG instillation, we tested the response of PBECs to BCG 


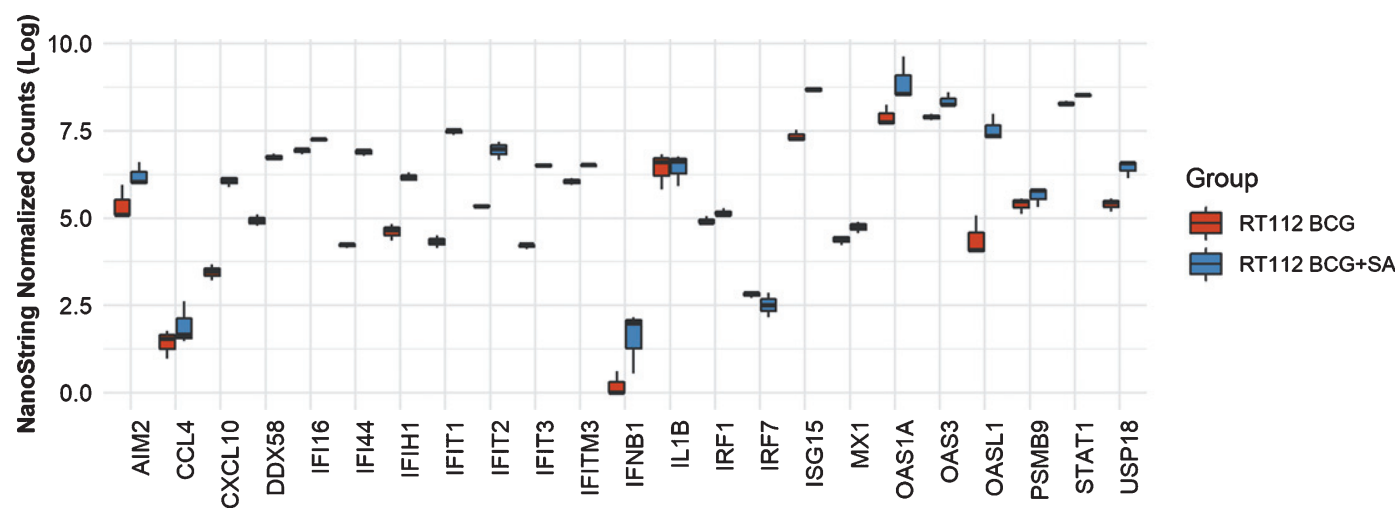

Fig. 4. Synergistic effect of BCG and STING agonist combination on induction of IFN1 genes in RT112 bladder cancer cells. NanoString platform-based gene expression profiling of RT112 bladder cancer cells treated with BCG and BCG+STING agonist. A custom IFN gene panel was used to determine expression changes at $6 \mathrm{~h}$ post treatment. RNA isolated from three independent experiments was used for gene expression analysis. NanoString data was normalized using nSolver software. Kruskal-Wallis test was applied to determine statistically significant $(p<0.05)$ expression differences between treatments.

$5 a$

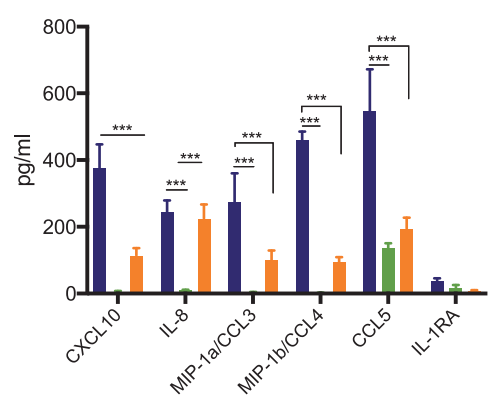

$5 b$

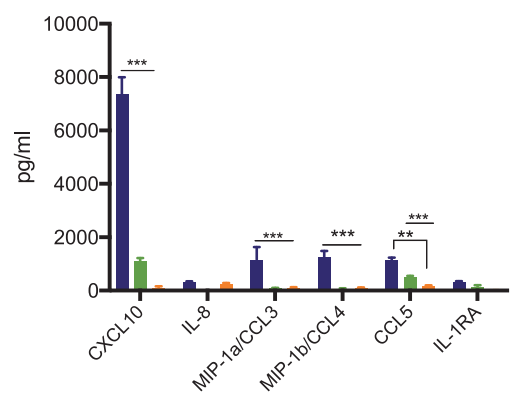

$5 c$

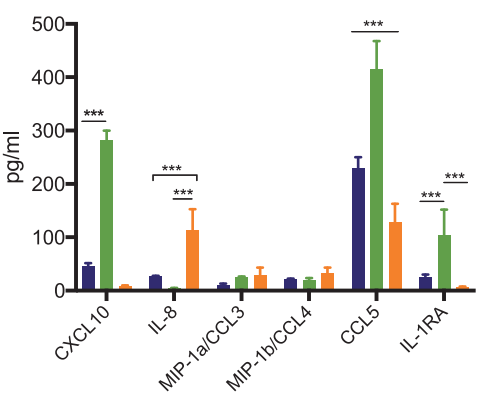

Fig. 5. Synergistic effect of BCG and STING agonist combination on secretion of immune cell recruiting chemokines. Synergistic effect of STING agonist and BCG in THP1, THP-1 NLRP3 KO and THP-1 dual STING KO, on induction of IFN induced chemokines at $24 \mathrm{~h}$ post BCG (5a), STING agonist BCG+STING agonist (5b) and STING agonist (5c), treatment. Cytokine data was analyzed using Graphpad Prism (8.0) software. Statistically significant differences were determined using a two-way repeated measure ANOVA followed by Tukey's post test. $* p=0.01, * * p=0.001, * * * p=0.0001$. Treatments were performed in triplicate. Data represent results from two independent experiments.

with or without STING agonist. Addition of STING agonist led to a significant increase in CXCL10, IL8 and IL-6 secretion at 24 post treatment, compared to BCG alone (Fig. 6). However, unlike THP-1 cells, levels of CCL5, MIP-1 and MIP-1b cytokines did not increase in the PBECs under any treatment condition (data not shown).

\section{DISCUSSION}

Since its initial use in 1976 [16], BCG represents not only the most effective immunotherapy for treatment of NMIBC [17] but also the best example of immune based cancer therapies. Although there continues to be a cumulative impact of several studies, focused on BCG associated response variations and biomarkers of response, on significantly advanc- ing our understanding of its anti-cancer effects, the precise mode of BCG action is still undefined and optimal management of NMIBC remains elusive.

Innate immune modulators as sensitizers to conventional therapies, have gained significant attention following the accumulation of evidence from several studies that show the positive association between favourable treatment outcomes and presence of IFNI induced gene signature in the pre-treatment tumours [8]. In the context of immune cells, CXCL10 production following IFN1 activation primarily depends on the status of STING pathway in BATF3+ DCs, which cross prime CD8+ T cells in the tumour draining lymph nodes and mobilizes them to the tumour site [12]. The STING pathway intersects with multiple cellular cytosolic nucleic acid sensing pathways that ultimately lead to IFN induced chemokine induc- 


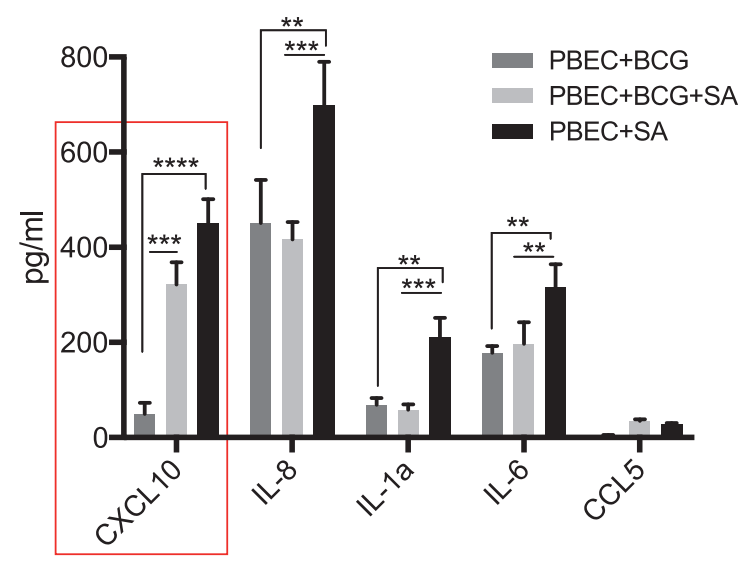

Fig. 6. Synergistic effect of BCG and STING agonist combination on secretion of immune cell recruiting chemokines by primary bladder epithelial cells (PBEC). Cytokine levels 24 post treatment of PBECs with BCG with or without STING agonist, were measured using multiplex-cytokine analysis of triplicates. Cytokine data was analyzed using Graphpad Prism (8.0) software. Statistically significant differences were determined using a two-way repeated measure ANOVA followed by Tukey's post-test. ${ }^{*} p=0.01,{ }^{* *} p=0.001,{ }^{* * *} p=0.0001$. Treatments were performed in triplicate. Data represent results from two independent experiments.

tion and therefore has recently re-gained attention in development of immunomodulatory cancer therapeutics [9-11].

In the current study we focussed on evaluating the effect of BCG and STING agonist combination, primarily using cell line models representing human monocytes, the bladder cancer cell line RT112 and primary cells (PBECs). We observed greater than 20-fold increase in secreted CXCL10 and others associated chemokines as a result of synergistic effect of BCG and STING agonist. Our in vitro findings provide compelling rationale for further pre-clinical and clinical studies on evaluating the addition of STING agonists or approaches such as oncolytic viruses that can activate STING pathway in the bladder resident immune cells to further increase in chemotaxis of CD8+ TILs.

Several previous studies have reported the urinary cytokine profiles soon after BCG instillation. The most consistent of these surrogate chemokines of IFN activation are CXCL10, IFN $\gamma$, IL-1, IL-6, IL10 , IL-2, TNF $\alpha$ and IL-12 [18, 19]. Urinary levels of these chemokines constitute the primary indicators of the magnitude of IFN activation and/or immune cell recruitment. Post BCG treatment levels of urinary or plasma CXCL10 and IL-8 could thus guide the addition of STING pathway activators in enhancing the levels of these chemokines. These findings not only depict the use of specific urinary cytokines as promising predictors of response, but also strongly indicate that the ability for BCG to induce these cytokines may be dependent on the bladder local immune cell composition at the time of first instillation.

Antigen presenting cells such as DCs (20\%) and macrophages $(40 \%)$ form the majority of immune cells in bladder mucosa. Other cell types include mast cells, CD4+ T cells, $\gamma / \delta+T$ cells and innate immune cells such as NK cells, monoctyes and eosinophils [20]. The resident immune cells including residual cancer cells at the time of treatment initiation, especially in cases of Tis, are at the forefront of response BCG. Thus, post BCG (microbial) challenge, macrophages and DCs being the primary cell targets for mycobacteria, are key to driving the chemokine led influx of neutrophils, monocytes and other immune cells from the circulation. One possible explanation for reduced BCG activity could be the higher infiltration of suppressive tumour associated macrophages (M2). Indeed higher proportions of M2 macrophages have been identified in the tumours from BCG non-responding patients [19, 21]. Moreover, alternatively activated M2 polarized macrophages do not produce CXCL10 or inhibit it via IL-4 and IL-10 [22]. Given the increased prevalence of bladder cancer in older individuals, this becomes even more critical since "inflammaging" could lead to higher M2 like tissue resident cells in the bladder [23].

Histopathological analysis of post BCG treatment bladder biopsies have confirmed epithelial denudation or exfoliation, which is critical to tumour antigen recognition by the DCs for cross priming to CD8+ $\mathrm{T}$ cells [24]. Antigen cross presentation via these antigen presenting cells presumably occurs either through trafficking via local lymph nodes or crosspriming to pre-existing $\mathrm{T}$ cells in the tumour immune microenvironment and could also play a role in response and/or recurrence [25].

We propose a model for the mode of action of BCG in bladder cancer wherein the STING pathway is integral to improving response via synergistically contributing to CXCL10 production (Fig. 1). Although difficult to demonstrate the causal link, a major cellular population that internalizes BCG at the time of intravesical treatment, is the epithelial lining which is constituted by non-malignant urothelial cells. This uptake is followed by tissue resident cell types including macrophages, dendritic cells and mast cells. Indeed, residual cancer cells (with variability in mutational status) also internal- 
ize BCG and exhibit downstream responses as shown by several previous studies and our current study. However, it is mechanistically challenging to prove these speculations given the genomic heterogeneity and age-related differences frequently observed in human tumours. Furthermore, mouse models are not accurate surrogates for studying immune cell make up given the lack of genomic heterogeneity and agerelated differences between the two species.

Immunomodulation via activation of innate immune sensing mechanisms such as STING pathway could be an effective synergistic therapy for BCG un-responsive patients. Oncolytic viruses, which function to activate cytosolic nucleic acid sensing pathways such as the cGAS-STING pathway, provide another promising strategy. In the context of cancer cells, excessive activation of IFN1 can lead to cellular senescence via activation of STING pathway and thus IFN stimulating agents must be used carefully such that progression to aggressive disease is avoided [26]. Similarly, in the context of immune cells, excessive IFN activation can lead to cytokine release syndrome with release of IL-6, IFN $\gamma$ and CXCL10, and associated adverse effects.

Several additional local therapies are being studied in patients with refractory disease states [27], [28]. Local delivery of exogenous interferon alpha (IFN $\alpha$ ) or via adenoviral mediated delivery of IFN $\alpha$ $2 \mathrm{~b}$ was the focus of several previous clinical trials in NMIBC [17]. Failure in response was primarily attributed to the transient effect of IFN $\alpha$ as a result of its short half-life. Based on the finding that prior BCG exposure associates with an increased response [29], the PRIME trial (NCT02326168) is investigating the prime boost concept of pre-sensitizing with $\mathrm{BCG}$ prior to induction treatment.

In addition to combining BCG with IFN $\alpha 2 b$, several other approaches have been tested, the most recent being the Listeria monocytogenes-listeriolysin gene integrated into BCG, VPM1002BC that imparts increased immunogenicity of BCG. Interestingly, VPM1002 leads to IL-1 $\beta$ and IL-18 production via activating the AIM2 inflammasome and STING pathway but not the NLRP3 inflammasome pathway. In line with this, given the success of PD-1/PD-L1 targeting immune checkpoint inhibitors in MIBC, several ongoing trials are now evaluating combining these agents with BCG therapies to improve response. Finally, Mycobacterium phlei cell wall nucleic acid (MCNA/MCC), demonstrated antineoplastic activity in high risk BCG un-responsive NMIBC and was evaluated in Phase III clinical trials and was proposed as an alternative to BCG [30]. Improved efficacy was reported following re-evaluation of the original publication when using more current definition of the true BCG unresponsive cohort [30, 31].

To circumvent for the reduced IFNI levels, similar to IFN $\alpha$, STING agonists could face the challenge of fast clearance, however, our study demonstrates a critical positive impact of activating the endogenous STING pathway in combination with BCG immunotherapy. Our in vitro study is the first to demonstrate the potential of complementing BCG with STING pathway activator to further increase TIL recruitment to prevent recurrence and progression.

Our study while novel, does have some limitations. BCG strain related differences in clinical outcomes, have been reported in several studies [32-34]. The Russian/Moscow strain of BCG (early strain with loss of RD1) was the first substrain established in 1924. The Tokyo, Tice and Connaught (late strains, evolved post 1925) substrains of BCG have a loss of both region of differentiation (RD) 1 and RD2 [35]. Interestingly, the Moscow strain exhibits superior immunogenicity compared to others such as Connaught, whereas a comparison between Connaught and Tice strain treated NMIBC patients revealed significantly increased recurrence free interval in the former only in induction treatments $[33,36]$. However, most of these studies have focused on the variable IL-8 levels induced by different BCG substrains in cancer cell lines.

Additional investigations evaluating the different strains of BCG would indeed be useful to determine if genetic drifting of the progeny [33] has resulted in a different capacity of various strains to affect the STING pathway. Given the scarcity in BCG availability, it is difficult to conduct a detailed investigation comparing these strains. Finally, since STING pathway activates PD-L1 expression [37], future trials should consider immune sensitization using STING agonists in combination with BCG for immune checkpoint blockade. An immediate next step could be pre-clinical investigations in syngeneic murine models of NMIBC to evaluate the efficacy of combinatorial BCG and STING pathway activators.

Our study included primary bladder epithelial cells, a novel perspective, that indicated that these cells secreted significantly higher IL- 8 and CXCL10 upon stimulation by STING agonist alone compared to BCG. Despite these limitations, findings from our study and culmination of several reports indicating that the activation of innate immune response to sensitize tumours to any treatment, warrant further 
evaluation in pre-clinical and clinical scenarios as a promising avenue for improving response to $\mathrm{BCG}$ in NMIBC patients.

\section{AUTHOR CONTRIBUTIONS AND ACKNOWLEDGMENTS}

MK, DRS and AM conceptualized and wrote the manuscript. MK and SC conducted the experiments. $\mathrm{SN}$ reviewed and performed the graphic design of schematic showing conceptual model of BCG mode of action and the potential of STING pathway activators. TV reviewed and conducted the statistical analysis of NanoString data. This study was funded by SEAMO Innovation funding support to DRS and MK.

\section{CONFLICT OF INTEREST}

The authors have no conflict of interest to report.

\section{SUPPLEMENTARY MATERIAL}

The supplementary material is available in the electronic version of this article: http://dx.doi.org/ 10.3233/BLC-190228.

\section{REFERENCES}

[1] Kamat AM, et al. Predicting Response to Intravesical Bacillus Calmette-Guérin Immunotherapy: Are We There Yet? A Systematic Review, Eur Urol. 2018;73(5):738-48.

[2] Song D, Powles T, Shi L, Zhang L, Ingersoll MA, Lu Y, Bladder cancer, a unique model to understand cancer immunity and develop immunotherapy approaches, 2019.

[3] Watson RO, et al. The Cytosolic Sensor cGAS Detects Mycobacterium tuberculosis DNA to Induce Type I Interferons and Activate Autophagy, Cell Host Microbe. 2015; 17(6):811-9.

[4] Cadena AM, Flynn JL, Fortune M. The Importance of First Impressions: Early Events in Mycobacterium. 2016; 7(2):1-9.

[5] Cheng Y, Schorey JS. Mycobacterium tuberculosis induced IFN- $\beta$ production requires cytosolic DNA and RNA sensing pathways, 2018, pp. 1-17.

[6] Gröschel MI, et al. Recombinant BCG Expressing ESX-1 of Mycobacterium marinum Combines Low Virulence with Cytosolic Immune Signaling and Improved TB Protection. Cell Rep. 2017;18(11):2752-65.

[7] Van Dis E, et al. STING-Activating Adjuvants Elicit a Th17 Immune Response and Protect against Mycobacterium tuberculosis Infection. Cell Rep. 2018;23(5):1435-47.

[8] Galon J. Approaches to treat immune hot, altered and cold tumours with combination immunotherapies. Nat Rev Drug Discov. 2019;18:10-13.
[9] Curran E, et al. STING Pathway Activation Stimulates Potent Immunity against Acute Myeloid Leukemia. Cell Rep. 2016;15(11):2357-66.

[10] Fu J, et al. STING agonist formulated cancer vaccines can cure established tumors resistant to PD-1 blockade. 2015;7(283).

[11] Ghaffari A, et al. STING agonist therapy in combination with PD-1 immune checkpoint blockade enhances response to carboplatin chemotherapy in high-grade serous ovarian cancer. Br J Cancer, 2018.

[12] Ager CR, Reilley MJ, Nicholas C, Bartkowiak T, Jaiswal AR, Curran MA. Intratumoral STING Activation with Tcell Checkpoint Modulation Generates Systemic Antitumor Immunity. Cancer Immunol Res. 2017;5(8):676-84.

[13] Kativhu CL, Libraty DH. A Model to Explain How the Bacille Calmette Guérin (BCG) Vaccine Drives Interleukin12 Production in Neonates. 2016;1:1-12.

[14] Koti M, et al. A distinct pre-existing inflammatory tumour microenvironment is associated with chemotherapy resistance in high-grade serous epithelial ovarian cancer. $\mathrm{Br} \mathbf{J}$ Cancer. 2015;112(7):1215-22.

[15] Au KK, et al. Gynecologic Oncology CXCL10 alters the tumour immune microenvironment and disease progression in a syngeneic murine model of high-grade serous ovarian cancer. Gynecol Oncol. 2017; 4-13.

[16] Morales A, Eidinger D, Bruce AW. Intracavitary Bacillus Calmette-Guerin in the treatment of superficial bladder tumors. J Urol. 1976;116(2):180-3.

[17] Arh S, Shepherd E, Nr B, Arh S, Shepherd E, Nr B. Intravesical Bacillus Calmette-Guérin with interferonalpha versus intravesical Bacillus Calmette-Guérin for treating non- muscle-invasive bladder cancer (Review), no. 3, 2017.

[18] Salmasi A, et al. Urinary cytokine profile to predict response to intravesical BCG with or without HS-410 therapy in patients with non-muscle invasive bladder cancer, 2018.

[19] Zuiverloon TC, Nieuweboer AJ, Vekony H, Kirkels WJ, Bangma CH, Zwarthoff EC. Markers predicting response to bacillus Calmette-Guerin immunotherapy in high-risk bladder cancer patients: A systematic review. Eur Urol. 2012;61(1):128-45.

[20] Hayes BW, Abraham SN. Innate Immune Responses to Bladder Infection, 2016, pp. 1-8.

[21] Ingersoll MA, Li X, Inman BA, Greiner JW, Black PC. and Translating Basic Science into the Clinic for Bladder Cancer Development of Bladder Cancer. 2018;4:429-40.

[22] Mantovani A, Sica A, Sozzani S, Allavena P, Vecchi A, Locati M. The chemokine system in diverse forms of macrophage activation and polarization. 2004;25(12).

[23] Van Beek AA, Van Den Bossche J, Mastroberardino PG, De Winther MPJ, Leenen PJM. Metabolic Alterations in Aging Macrophages: Ingredients for In fl ammaging? Trends Immunol., 2018, pp. 1-15.

[24] Luque RJ, Mazzucchelli R, Scarpelli M, Montironi R. Changes produced in the urothelium by traditional and newer therapeutic procedures for bladder cancer, no. May, 2002, pp. 641-647.

[25] Dockrell HM, Smith SG. What Have We Learnt about BCG Vaccination in the Last 20 Years? 2017;8:1-10.

[26] Gui X, et al. primordial function of the cGAS pathway, Nature.

[27] Nykopp TK, Batista J, Mannas M, Black PC. Current Clinical Trials in Non-muscle Invasive Bladder Cancer, 2018. 
[28] Li R, Spiess PE, Kamat AM. Treatment Options for Patients with Recurrent Tumors After BCG Therapy: Are We Ignoring the Obvious? Eur Urol. 2018, pp. 0-3.

[29] Biot C, et al. Preexisting BCG-Specific T Cells Improve Intravesical Immunotherapy for Bladder Cancer. Sci Transl Med. 2012;4(137):137ra72 LP-137ra72.

[30] Morales A, Cohen Z. Mycobacterium phlei cell wall-nucleic acid complex in the treatment of nonmuscle invasive bladder cancer unresponsive to bacillus Calmette-Guerin, Expert Opin. Biol Ther. 2016;16(2):273-83.

[31] Li R, Amrhein J, Cohen Z, Champagne M, Kamat AM. Efficacy of Mycobacterium Phlei Cell Wall-Nucleic Acid Complex (MCNA) in BCG-Unresponsive Patients. 2017;3:65-71.

[32] Anderson EJ, et al. The influence of BCG vaccine strain on mycobacteria-specific and non-specific immune responses in a prospective cohort of infants in Uganda, Vaccine. 2012;30(12):2083-9.
[33] Noon AP, Kulkarni GS. ' rin (BCG) Strains Are Equal, but Some All Bacillus Calmette-Gue BCG Strains Are More Equal Than Others. Eur Urol. 2014;66(4):689-91.

[34] Gan C, Mostafid H, Khan MS, Lewis DJM. REVIEWS BCG immunotherapy for bladder cancer - the effects of substrain differences, 2013, pp. 1-9.

[35] Secanella-fandos S, Luquin M, Julián E. Connaught and Russian showed the highest direct antitumoral effects among different BCG substrains, JURO, 2012.

[36] Witjes JA, et al. The efficacy of BCG TICE and BCG Connaught in a cohort of 2, 099 patients with T1G3 non - muscle-invasive bladder cancer. Urol Oncol Semin Orig Investig. 2016;34(11):484.e19-484.e25.

[37] Lemos H, et al. STING Promotes the Growth of Tumors Characterized by Low Antigenicity via IDO Activation. 2016;6(8). 